\title{
Application of extended free net adjustment constraints in two-step analysis of deformation network
}

\author{
Gilad Even-Tzur ${ }^{1} \cdot$ Lior Shahar ${ }^{1}$
}

Received: 18 December 2014/Accepted: 27 April 2015/Published online: 12 May 2015

(c) Akadémiai Kiadó 2015

\begin{abstract}
Two-step analysis of deformation network enables the extraction of geodynamical quantities from geodetic measurements campaigns in two steps. In the first step the measurements' mathematical model is realized for each monitoring campaign and in the second step the deformation model is examined. The mathematical model is usually conceived as being absolutely correct, while the validity of the deformation model and its system noise is frequently limited. The deformation model is commonly presented by kinematic model although dynamic model might describes the geophysical reality more accurately. Dynamic model is usually characterized by nonlinearity, which makes difficult the analysis of deformations in relative to a stable datum. Therefore, many of the control networks that are used for deformation monitoring and are measured by geodetic measurements are currently defined by kinematic models. In monitoring networks, global effects can impair the data processing and the deformation analysis and cause the deviation of the network solution. Extended free net adjustment constraints is a mathematical method that effectively coping with global effects. An extended solution of geodetic network for deformation monitoring includes the solution of extended parameters, in addition to those received in a standard solution. Such a solution enables to sterilize the geodetic measurements from their datum definition content in the first step, and extract the deterministic movement in the second step. The paper shows the great potential of using combination of the extended free net adjustment constrains and the two-step analysis of deformation networks.
\end{abstract}

Keywords Geodetic monitoring networks · Deformation monitoring · Datum · Dynamic model

Gilad Even-Tzur

eventzur@technion.ac.il

1 Division of Mapping and Geo-Information Engineering, Faculty of Civil and Environmental

Engineering, Technion - Israel Institute of Technology, 32000 Haifa, Israel 


\section{Introduction}

Geodetic monitoring networks are used to extract geodynamical quantities such as displacements, velocities and strains. These quantities are defined and solved relative to a reference frame, datum. The free network adjustment techniques developed by Meissl in the 1960s plays a major role in the analysis of deformation networks (e.g. Meissl 1969). It has been employed extensively in time dependent analysis of geodetic networks and has been used as a means for solving the inherent datum problem of the geodetic network. In free network, datum is defined by the selection of a subclass of its points. The selection of the datum points is subjective and depends on a number of considerations such as, for example, the destination of the network, the specific type of measurements, the geotechnical properties of the ground, the way the points are structured and more (Papo 1999).

Two model types are involved in deformation analysis: the mathematical model that represents the geodetic measurements, and the deformation model. The mathematical model is usually conceived as being absolutely correct, while the measurements are regarded as quantities corrupted by measurement noise. The deformation model should describe the geophysical reality, but the validity of the geophysical model and its system noise is frequently limited. The deformation model is usually presented today by defining a kinematic model (e.g. Qu et al. 2014; Brockmann et al. 2012; Árnadóttir et al. 2006; Fu et al. 2002). Using kinematic model to describe complicated geophysical environs can lead to the partial estimation of the deterministic dynamics, which characterizes the entire network. By using dynamic model we might represent the geophysical reality more accurately. Dynamic model interprets the movement parameters of the kinematic model, and provides a mathematical definition that more accurately describes the geophysical reality (Dong et al. 1998; Papo and Perelmuter 1991). These interpretations are typically characterized by non-linearity and are not suitable for a Cartesian coordinates system. Therefore, it is impossible to define a stable datum using this kind of model. Because of these complexities, many of the control networks that are used for deformation monitoring and are measured by geodetic measurements are currently defined by kinematic models.

Extended free net adjustment constraints (Papo 1985, 1986; Even-Tzur 2011) is a mathematical method enables the solution of parameters that describe the network's global deterministic behavior in addition to the regular adjusted parameters, which are point-coordinate corrections. The method enables the use of a dynamic model, while the estimation of global movement as residuals is minimized (Papo 1986). The research makes use of extended free net adjustment constraints incorporated into the two-step analysis of dynamical networks (Papo and Perelmuter 1993; Even-Tzur 2004). The two-step analysis method is based on a first step of independent adjustment of every measurement campaign to a set of network points coordinates, followed by a second step in which we use the coordinates as pseudo-observations for adjusting a set of kinematical and dynamic parameters. Analysis of deformation in two consecutive steps is to be preferred over a single-step procedure because it provides a clear-cut separation in terms of models and estimation (Koch and Papo 2003).

\section{Theoretical background}

\subsection{Two-step deformation analysis}

The relationship between a vector of $n$ measurements $\ell$, which contains the differences between observed and computed measurements, and a vector of $u$ parameters $\mathbf{w}$, which 
contains the corrections to the approximate values of the parameters $\mathbf{w}^{0}$, can be expressed by a set of observation equations given as:

$$
\ell=\mathbf{C w}-\mathbf{v}
$$

$\mathbf{C}$ denotes Jacobian matrix (the design matrix) and $\mathbf{v}$ is the vector of measurement corrections. It may be assumed that $\mathbf{C}$ is a column rank deficient matrix due to the need for datum definition.

The parameters $\mathbf{w}$ are calculated under the minimum condition $\mathbf{v}^{T} \mathbf{P v}$, where $\mathbf{P}$ is the weight matrix of the observations.

As the vector of parameters $\mathbf{w}$ can be expressed as a linear function of a vector of parameters $\mathbf{s}$, another set of equations may be created:

$$
\mathbf{w}=\mathbf{R s}-\mathbf{z}
$$

where $\mathbf{R}$ denotes the Jacobian matrix and is usually a full column rank matrix, $\mathbf{z}$ is the model noise vector. The parameters $\mathbf{S}$ are calculated under the minimum condition $\mathbf{z}^{T} \mathbf{P}_{\mathrm{Z}} \mathbf{z}$, where $\mathbf{P}_{\mathrm{z}}$ is the weight matrix of the observations in the second model.

This approach for solving $\mathbf{s}$ indirectly from the measurements vector $\ell$ while using a vector of pseudo-measurements $\mathbf{w}$ is referred to as a two-step analysis (Papo and Perelmuter 1993; Even-Tzur 2004), where in the first step w is solved using the measurement vector $\ell$, and in the second step $\mathbf{w}$ is used as pseudo-measurements for solving the parameter vector $\mathbf{s}$.

Study of deformations, which is based on the use of geodetic measurements, can be done efficiently and conveniently by using the two-step analysis method. When using this method, the first step includes the adjustment of measurement campaigns to an independent set of network points coordinates. Therefore, this step does not include any reference to network changes that have occurred during monitoring. In addition, the adjusted coordinates and their covariance matrix allow to perform quality control for each monitoring campaign as well as a comprehensive assessment of the quality of the network and its ability to distinguish deformations. The interim results of the first step serve as a convenient basis for detecting gross measurement errors, problematic measurement campaigns, or problematic network points.

During the second step, the coordinates obtained in the first step serve as pseudoobservations, which are used to adjust an approximate deformation parameters. Estimation of the deformation parameters directly from the geodetic measurements may lead to undesirable results. System noise due to inadequacies of the physical model may cause severe distortions of the parameter estimates (Even-Tzur 2004).

The two-step method is defined with modularity that contributes to future monitoring. While the simultaneous adjustment requires the measurements data for each campaign, the two-step method requires only some calculated parameters of the first step: the adjusted coordinates set and their covariance matrix, the sum squares of residuals and the degree of freedom of each campaign.

\subsection{Extended free network adjustment constraints: EFNAC}

In addition to the adjusted parameters, an extended solution estimates parameters that describe the network's global behavior. To review the principle of the Extended Free Network Adjustment Constraints let us discuss, as an example, the vector of adjusted parameters $\mathbf{w}$ as presented in (Eq. 1). The vector $\mathbf{w}$ is partitioned into global and local 
components through the introduction of a vector of parameters $\mathbf{y}$ (Papo 1985). Vector $\mathbf{y}$ describes the global components and contains $f$ parameters. Vector $\mathbf{y}$ is limited by the inequality, $\mathrm{f}<\mathrm{u}-\mathrm{d}$, where $d$ is the size of the datum defect.

Let us denote $\mathbf{x}$ as the vector of corrections to the approximate values of the parameters $\mathbf{x}^{0}$ and $\mathbf{y}$ as corrections to the preliminary value $\mathbf{y}^{0}$.

The elements of $\mathbf{y}$ can be related as the parameters of a transformation (mapping function) between the two vectors $\mathbf{x}$ and $\mathbf{w}$. The differences between $\mathbf{x}$ and $\mathbf{w}$ are the characteristics represented by the transformation and the parameters $\mathbf{y}$. Therefore $\mathbf{w}$ can be presented as,

$$
\mathbf{w}=\mathbf{D} \mathbf{x}+\mathbf{F y}
$$

where $\mathbf{D}=\partial \mathbf{w} / \partial \mathbf{x}$ is a $u \times u$ full rank matrix and $\mathbf{F}=\partial \mathbf{w} / \partial \mathbf{y}$ is a $u \times f$ matrix of full column rank.

Therefore, the adjusted parameters $\mathbf{x}^{a}$ and $\mathbf{y}^{a}$ are equal to $\mathbf{x}^{a}=\mathbf{x}^{0}+\mathbf{x}$ and $\mathbf{y}^{a}=\mathbf{y}^{0}+\mathbf{y}$. Now, the observation Eq. (1) are rewritten as,

$$
\ell+\mathbf{v}=\mathbf{C}(\mathbf{D x}+\mathbf{F y})=\mathbf{C}(\mathbf{D}, \mathbf{F})\left[\begin{array}{l}
\mathbf{x} \\
\mathbf{y}
\end{array}\right]=(\mathbf{A}, \mathbf{B})\left[\begin{array}{l}
\mathbf{x} \\
\mathbf{y}
\end{array}\right]
$$

The rank of $(\mathbf{D}, \mathbf{F})$ is $u$ (since $(\mathbf{D}, \mathbf{F})$ is a $u \times(u+f)$ matrix). The rank of $(\mathbf{A}, \mathbf{B})$ is $u$ - $d$, the same as the column rank of $\mathbf{C}$ (since the rank of $\mathbf{C}(\mathbf{D}, \mathbf{F})$ is equal to the rank of $\mathbf{C}$ ). This means that the number of estimable parameters in the extended system is $u-d$. The size of the null space of $(\mathbf{A}, \mathbf{B})$ is thus $d+f$, meaning that $d+f$ linear conditions between the parameters are necessary to obtain a minimally constrained solution. The solution of $\mathbf{x}$ and $\mathbf{y}$ and their cofactor matrix by means of EFNAC is presented in detail by Koch and Papo (1985), Papo (1986), Even-Tzur (2011) and Shahar and Even-Tzur (2012).

\section{Two-step analysis combined EFNAC}

\subsection{Applying extended free network adjustment in the first step}

Geodetic measurements can define the inner geometry of the points in the network, but they are incapable of completely determining its datum. A datum consists of three components: origin, orientation, and scale. Various geodetic measurements define various components of a datum. A datum defect expresses the partial absence of a reference system and enables an infinite number of trivial constrained solutions. The defect of a 3D geodetic network, for example, is three when the definition of origin is missing and it can grow to seven when the definition of the rotations and scale are missing as well. Geodetic measurements contain part of the datum definition. For example, measured distances in a network can define its datum parameter of scale, while at the same time the distances contribute to defining the relative positions of points in the network. The remaining datum parameters identified with the datum defect of the observational system are defined by imposing an equal number of linear constraints on the estimated coordinate corrections (Koch 1999).

Free net adjustment has been employed extensively in time dependent analysis (4D) of geodetic networks and has been used as a means for solving the inherent datum problem of the geodetic network (Papo 1985). In 4D geodetic network, the part of the datum definition determined by the geodetic measurements may not remain consistent. In each monitoring 
campaign, the network may be measured by different type of instruments and different measuring methods. Even when using the same instrument and the same measuring methods we cannot ensure that the part of the datum definition determined by the measurements is the same in each monitoring campaign and remains stable over time.

In monitoring geodetic networks, the deformation parameters can be estimable only if the datum of the network has not been changed between measurement epochs. The measurements of a monitoring network must be adjusted so that the datum will remain undisturbed. However, as was mentioned before, there are geodetic measurements that contain datum definition components. When an appropriate measure is not taken to prevent the inclusion of these components in the adjustment of a 4D network, the result might be an inevitable mixture of the deformation parameters and the datum components of the measurements.

The proper way of avoiding disturbance to the datum components of the measurements is to sterilize the geodetic measurements from their datum definition content. The conventional datum of the geodetic network in each monitoring campaign is then defined in its entirety by the preliminary coordinates of its points and the linear constraints imposed on the corrections to those coordinates. For example, the proper way to incorporate distance measurements in a network is to filter out their datum definition of scale and utilize only the sterilized (datumless) quantities in the adjustment. The process of withholding the geodetic measurements datum definition content in the adjustment of the network can be achieved by using extended free network adjustment constrains (Papo 1985; Even-Tzur 2011).

Vector w (Eq. 3) is partitioned into local and global components, while in the first step the vector of global parameters $\mathbf{y}$ represents the contribution of the measurements to the global component of the point coordinates and $\mathbf{x}$ represents sterilized coordinates. Vector $\mathbf{x}$ represents a coordinate were the global content of the measurements has been withheld.

In each monitoring campaign the geodetic measurements were adjusted into a network using the extended free network adjustment constraints. The measurements are stripped from there datum contents and the datumless measurements are used to define the datum by preliminary coordinates and linear constraints that remain constant for all monitoring campaigns, as well as defining the position of the network points. The solution of the network points' position and their variance-covariance matrix are used as pseudo-measurements for the solution of the second step. In order to ensure the same units system throughout the all stages of the solution it is mandatory that the units system be uniform for the measurements as well as for the preliminary coordinates.

The implementation of extended free network adjustment in a leveling network so as to strip the measured coordinates from their scale content is presented in Shahar and EvenTzur (2014a). The method also successfully applied in trilateration network, so as to strip the datum content of scale (Even-Tzur 2011) and in a GPS network, so as to strip the datum content of scale and orientations from the measured coordinates (Even-Tzur and Reinking 2013) in order to estimate the velocity field of monitoring network.

\subsection{Applying extended free network adjustment in the second step}

Estimation of the second step solution using EFNAC enables the separation of the network's deterministic dynamics and local deformations. The deterministic component interprets the global dynamic behavior in the network. The local deformation is expressed by residuals of the kinematic components, usually of the velocity field. Therefore, in the second step s (Eq. 2) is partitioned into global and local components, while $\mathbf{y}$ represents the parameters that define the dynamic interpretation of the network points' position as 
function of time. Vector $\mathbf{x}$ represents the location of points as function of time were the global dynamic interpretation has been withheld.

As mentioned, geodynamical quantities such as displacements, velocities and strains can be estimable only if the datum of the network has not been changed over the measurement epochs. When discussing deformation networks, a datum is defined as a subset of network points. Therefore, the selection of the datum points involves a number of considerations. The chosen network points must be consistent and stable over time. Consequently, the datum points must be located in a geologically stable and homogeneous area, in order to ensure the inner stability over time of all the datum points.

When the monitoring area has a global non-linear dynamics, it is not possible to determine a stable group of points which can serve as datum due to deformation among the datum points. In such a case, the definition of a datum may become possible through the description of the datum points using a dynamic model that describes its internal deterministic deformation. Such a solution is possible by using EFNAC. The extended solution extracts the deterministic component from the kinematic coordinates of the datum points and enables the analysis of its residuals (as the solution sub-vectors $\mathbf{x}$, see Eq. 4). Passing a partial congruency test of the sub-vector $\mathbf{x}$ indicates the stability of the examined datum, which means an absence of significant local inherent deformation in the datum. Not passing a partial congruency test, iterative process can be apply to remove one datum point at $\mathrm{s}$ time until the congruency test passes. In addition to the statistical congruency test, geological logic should be taken into account when selecting the datum points, which justifies internal stability excluding the deterministic dynamics. The method for datum definition in areas with non-linear dynamics by using EFNAC enables to define datum in an unconventional manner, it let us to define datum with dynamic nature, dynamic datum. As classical datum, so dynamic datum should contain as many as possible points to insure stable, accurate and reliable definition for deformation monitoring.

The implementation of dynamic datum solution by means of EFNAC in leveling network is presented in Shahar and Even-Tzur (2014b).

\section{An example: infinite long strike-slip fault model}

Let us demonstrate the principles of the proposed method and emphasize its advantages on the infinite long strike-slip fault model in a uniform elastic half space (Cohen 1999) as an theoretic example. We elect this model because it describes a global non-linear behavior. Therefore, the relative velocities between monitoring points are non-linear. In this model, the velocity of a network point in parallel direction to the fault is defined by (Fig. 1):

$$
v_{x}=\pi^{-1} V \tan ^{-1} \frac{r}{L D}
$$

where $r$ represents the orthogonal distance between the point and the fault, $L D$ represents the locking depth of the fault and $V$ represents its velocity. The model presents by (5) is a dynamic model, it is actually a physical interpretation of the coordinate's velocity. Therefore, the coordinates of a point at a given time $t_{i}$ can be presented by:

$$
x_{i}=x_{0}+\pi^{-1} V \tan ^{-1} \frac{r}{L D} \Delta t_{i}
$$

when $x_{0}$ is the standard position at the standard time $t_{0}$ and $\Delta t_{i}=t_{i}-t_{0}$. The mathematical solution of the model required to determine part of the physical model parameters. In 


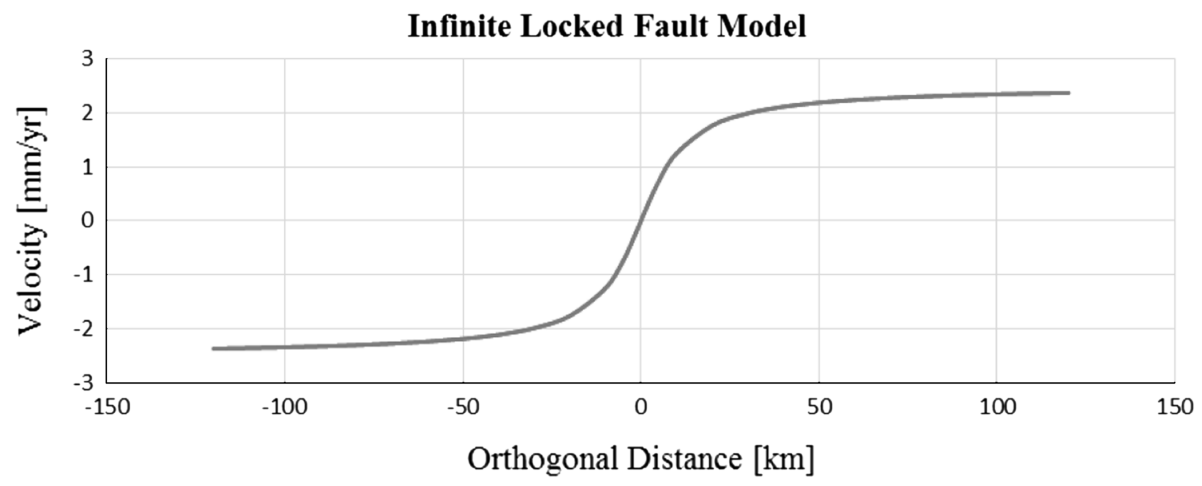

Fig. 1 Model of Infinite Locked Fault when the velocity of a point is a function of its ortogonal distance from the fault. In this example the fault is locked up to $L D=10 \mathrm{~km}$ with transform velocity of $V=5 \mathrm{~mm} /$ year

infinite locked fault model the fault location and the depth of locking must be determined to insure a stable mathematical solution (Shahar and Even-Tzur 2012).

The second step of deformation analysis uses the adjusted coordinates of the first step as pseudo-observation. This step revaluates parameters according to a movement model that includes the time dimension and enables revaluation of kinematic parameters, in addition to the standard network coordinates. While executing an extended result of the first step, the pseudo-observations are regarded as cleaned and are not affected by any datum elements defined by measurements. In the second step, an extended solution enables the separation of the network's deterministic movement and local deformations. The extended parameter which represents the deterministic movement is the transform velocity, therefore $\mathrm{y}=V$. The local deformation is expressed by the vector of residuals that is obtained from the solution of the second step. Hence, $\mathbf{D}$ is defined as identity matrix, $\mathbf{D}=\partial \mathbf{s} / \partial \mathbf{x}=\mathbf{I}$ and $\mathbf{F}$ as $\mathbf{F}=\partial \mathbf{s} / \partial \mathbf{y}=\partial \mathbf{s} / \partial \mathbf{V}$.

In monitoring network, which covers an area that behaves according to infinite locked fault model it would be very difficult to set a stable datum. The expected relative movements between points, however small, prevents the ability to define a stable datum. Nonetheless, there are two alternatives to defining a stable datum in this scenario. One alternative is to define a stable datum by points that are on one side of the fault and are located equidistant from the fault. No relative movements are expected between these points and therefore they remain stable and can be selected as datum. However, the probability of finding some points that meet this requirement is very small so this option is very limited and may not be applied in general cases. Another more practical option is to set datum base on points that are located far away from the fault. The relative movement between these points is expected to be small (see Fig. 1) and therefore it may be able to set stable datum based on these points. However, it is well known that positional accuracy of points in a free network depends heavily on the spatial distribution of its datum definition points. Positional accuracy of a point is inversely proportional to its distance from the mass center of the datum points (Papo 1999). Because the greatest interest is the investigation of movements of points close to the fault, selecting this datum can be a hindrance to the analysis of deformations. 
An extended solution of a control network with the extraction of the deterministic dynamics and defining the datum as dynamic datum enables us to set a datum based on large a number of points, regardless of changes in distance from the fault line.

\section{Summary and conclusion}

The paper presents a combination of two known concepts for solving geodetic control network an extended mathematical solution combined in two steps analysis of deformation networks. The advantages of the two-step method lies in the separation between the two steps. In the first step the unknown parameters, which are usually coordinates, are estimated for each monitoring campaign using field measurements. Each campaign is solved autonomously without consideration of the deformation model. This form of solution increases the ability for gross error detection and enables evaluation of the quality of the measurements of each monitoring campaign. In the second step, the coordinates are used as pseudo measurements in the solution of the deformation parameters. One can experiment with alternative deformation models or hypotheses until achieving satisfactory results.

An extended mathematical solution of geodetic network for deformation monitoring includes the solution of extended parameters, in addition to those estimated in a standard solution. The extended solution enables us to extract deterministic ingredient that defines the entire control network. This extraction differentiates between the network coordinates and the deterministic parameters that are liable to bias the solution. We distinguish between two types of global deterministic parameters, which are revalued as an inherent part of the standard solution of a control network: geometric parameters and physical parameters. The geometric parameters express the internal deformation of the standard network, while the physical parameters express the physical interpretation of some of the kinematic parameters that define the network model, usually including the velocity field.

The adjustment system for estimation of deformation parameters is defined according to a basic assumption regarding datum stabilization over time. This assumption, however, is not necessarily correct. The method used in this study minimizes the problematic nature of this assumption by differentiating and distinguishing between the measurements and the datum components, thus eliminating the possibly erroneous effects of the assumption. This method may contribute to improving the dealing with measurements and thus improving the ability of the control network to monitor deformations.

The mathematical estimation of parameter that describe geophysical reality requires the use of a dynamic model. The definition of a stable datum requires the extraction of the global physical parameters, which enables the definition of a datum that more closely reflects the geophysical reality of the control network and prevents systematic errors from spreading from the raw measurements to the system for the estimation of parameters. The extraction thus prevents systematic error from being erroneously interpreted as a deformation.

In conclusion, this paper wishes to emphasize the great advantage of using the extended free network adjustment constraints combined with two-step deformation analysis.

\section{References}

Árnadóttir T, Jiang W, Feigl KL, Geirsson H, Sturkell E (2006) Kinematic models of plate boundary deformation in southwest Iceland derived from GPS observations. J Geophys Res 111:B07402. doi:10. 1029/2005JB003907 
Brockmann E, Ineichen D, Marti U, Schaer S, Schlatter A, Villiger A (2012) Determination of tectonic movements in the Swiss Alps using GNSS and levelling. Int Assoc Geod Symp 136:689-695

Cohen SC (1999) Numerical models of crustal deformation in seismic zones. Adv Geophys 41:134-231

Dong D, Herring TA, King RW (1998) Estimating regional deformation from a combination of terrestrial geodetic data. J Geod 72:200-214

Even-Tzur G (2004) Variance factor estimation for two-step analysis of deformation networks. J Surv Eng 130(3):113-118

Even-Tzur G (2011) Deformation analysis by means of extended free network adjustment constraints. J Surv Eng 137(2):47-52

Even-Tzur G, Reinking J (2013) Velocity field across the Carmel fault calculated by extended free network adjustment constraints. J Appl Geod 7(2):75-82

Fu Y, Zhu W, Wang X, Duan W, Wu X, Jiao W (2002) Present-day crustal deformation in China relative to ITRF97 kinematic plate model. J Geod 76:216-225

Koch KR (1999) Parameter estimation and hypothesis testing in linear models, 2nd edn. Springer, Berlin

Koch KR, Papo H (1985) Erweiterte freie Netzausgleichung. ZFV 110(10):451-457

Koch KR, Papo H (2003) The Bayesian approach in two-step modeling of deformations. Allg Vermess Nachr 110(111):365-370

Meissl P (1969) Inner theory of errors of a cluster of points. In: Rinner K (ed) Annex F in systematic investigations of geodetic networks in space, pp 120-131

Papo H (1985) Extended free net adjustment constraints. Bull Géod 59(4):378-390

Papo H (1986) Extended free net adjustment constraints. NOAA technical report NOS 119 NGS 37, September 1986

Papo H (1999) Datum accuracy and its dependence on network geometry, Int. Meeting "Quo Vadis Geodesia...?" in honor of Prof. Erik Grafarend's 60th birthday, Stuttgart 3-4 November 1999

Papo H, Perelmuter A (1991) Dynamical modeling in deformation analysis. Manuscr Geod 18:295-300

Papo H, Perelmuter A (1993) Two-step analysis of dynamical networks. Manuscr Geod 18:422-430

Qu W, Lu Z, Zhang Q, Li Z, Peng J, Wang Q, Drummond J, Zhang M (2014) Kinematic model of crustal deformation of Fenwei basin, China based on GPS observations. J Geodyn 75:1-8

Shahar L, Even-Tzur G (2012) Extraction of the deterministic ingredient of a dynamic geodetic control network. J Geod Sci 2(1):68-75

Shahar L, Even-Tzur G (2014a) Improved analysis of vertical movements in the carmel fault region, Israel, by extended free net adjustment. Zeitschrift fur Geodaesie, Geoinformation und Landmanagement 139(2):115-124

Shahar L, Even-Tzur G (2014b) Definition of dynamic datum for deformation monitoring: carmel fault environs as a case study. J Surv Eng 140(2):04014002 